\title{
Data evaluation of BDS registers from airborne transponders
}

\author{
Jan Angelis \\ Department of Air Transport \\ Czech Technical University in Prague \\ Prague, Czech Republic \\ angelis.cz@gmail.com
}

\author{
Jiř́ Frei \\ Planning and Development Division \\ ANS CZ \\ Prague, Czech Republic \\ frei@ans.cz
}

\author{
Stanislav Pleninger \\ Department of Air Transport \\ Czech Technical University in Prague \\ Prague, Czech Republic \\ pleninger@fd.cvut.cz
}

\begin{abstract}
The purpose of this article is the evaluation of data from BDS registers of aircraft transponders with the focus to calculate wind speed and wind direction. First part of this article focusses on BDS registers and method of calculating wind values from data of secondary surveillance radars obtained from Air Navigation Services of the Czech Republic. Evaluation and comparison of the calculated data with the real values of the wind is stated in the second part of the article.
\end{abstract}

Key words: BDS registers, mode $S$, secondary surveillance radar, calculation of wind speed and wind direction

\section{INTRODUCTION}

Communication between aircrafts and Air Traffic Control (ATC) is via voice or data communications. One of the most frequent ways of data communication is data transmission through Secondary Surveillance Radars (SSR) using mode S which allows transmitting not only identification but even position or any additional information such as information about speed and course, height, yawing, meteorological data etc. Individual information is uploaded into BDS registers (Binary Data Store) in the onboard transponder, from where they can be transmitted through mode S transponder to SSR.

This article aims to create a method that can calculate values of altitude wind field - WSP (wind speed) and WDI (wind direction) from the data stored in BDS registers $44_{16}$. Calculated values of altitude wind field will be compared with the real values from aircrafts in BDS registers $44_{16}$, so we can evaluate the accuracy of method used for calculation altitude wind field. As another way how to evaluate calculated values of WSP and WDI will be chosen comparison with real value of wind from WAFC (World Area Forecast Center) and graphic comparison with meteorological maps from American archive of Plymouth State Weather Center.
Primary data for the calculation of WSP and WDI were obtained from ANS of the Czech Republic from secondary surveillance radars SSR Praha, Písek and Buchtův kopec.

\section{BDS REGISTERS}

\section{A. Definition of BDS registers}

The mode $S$ function is based on data transmission between interrogator (ground segment) and aircraft transponder. To ensure the data transfer between the transponder and interrogator, the requested data must be available from onboard equipment. The actual onboard data are stored inside SSR transponder depository divided into BDS registers.

There are 255 BDS registers where each register has a size of 56 bits and contains predetermined parameters. Assignment of particular parameters into single registers is in accordance with Annex 10 Volume III, Appendix to Chapter 5. Some of the registers are unallocated or reserved for future usage.[1]

Data transmission is usually initiated by ground segment by GICB protocol (Ground Initiated Comm-B), which allows to extract particular data (parameters) from selected register and transfer them as a response to query encrypted in Comm-B message (112 bits response with 56 bits message field) or in some cases in Comm-D message (112 bits response with 80 bits message field). Data from airborne equipment are automatically available for ATC units without the need of voice communication. [2]

\section{B. Definition of BDS registers for purposes of WSP, WDI calculation}

In SSR data obtained from ANS of the Czech Republic are included several BDS registers with relevant parameters. For purposes of calculation and evaluation of WSP and WDI are needed following BDS registers: 
1) BDS register $44_{16}$ - Meteorological routine air report: Register contains information about wind speed, wind direction, static air temperature, average static pressure, turbulence and humidity.

2) $B D S$ register $50_{16}$ - Track and turn report: Register contains information about roll angle, true track angle, ground speed, track angle rate and true airspeed.

3) $B D S$ register $60_{16}$ - Heading and speed report: Register contains information about magnetic heading, indicated airspeed, Mach's number, barometric altitude rate and inertial velocity.

Although every BDS registers contains several parameters, for the purposes of this article we need only some of them:

- BDS register $44_{16}$ - wind speed (WSP) and wind direction (WDI).

- BDS register $50_{16}$ - true track angle (TRK), ground speed (GSP) and true airspeed (TAS)

- BDS register $60_{16}$ - magnetic heading (MGH).

\section{FILTERING AND COMPUTING ALGORITHM}

\section{A. Filtering records}

Data from SSR are stored in format of text file and for their usage is used database software MySQL [3]. One day record is represented by 1.5 million rows and 27 columns in database structure. Rows represent single records from SSR and columns are relevant parameters from BDS registers.

On each data file in MySQL is used a filter algorithm which removes useless parameters. As a next step the algorithm removes records where the values of parameters TRK, GSP, MGH and TAS are missing, because the values of WSP and WDI cannot be calculated without them. The ratio of missing values of TRK, GSP, MGH, TAS parameters is about $3 \%$ records per 1 day.

After filtering records the data are reduced down only to parameters of date and time (DATUM), geographical longitude (E) and latitude (N), flight level (FL), WSP, WDI, TRK, GSP, $\mathrm{MGH}, \mathrm{TAS}$ and are ready for export.

\section{B. Calculating algorithm}

For calculation of WSP and WDI is used mathematical software MATLAB. The method of calculating WSP and WDI comes from the basic principle of triangle navigation scheme used in aeronautics and has a following form of equations:

$$
\begin{aligned}
& V_{x}=(-T A S * \sin (M G H+D E C))+G S P * \sin (T R K) \\
& V_{\mathrm{y}}=(-T A S * \cos (M G H+D E C))+G S P * \cos (T R K) \\
& W S P_{-} v y p=\sqrt{\left(V_{x}^{2}+V_{y}^{2}\right)} \\
& W D I \_v y p=\left(270-\operatorname{arctg} 2\left(V_{y} / V_{x}\right)\right) \bmod 360
\end{aligned}
$$

In Equation (3.1), (3.2) variable DEC represents magnetic variation and in Equation (3.4) the variable mod 360 represents modulo function (exact division). Calculated values of parameters WSP and WDI are called as WSP_vyp and WDI vyp so there won't be any mismatch with WSP or WDI from $\overline{B D S}$ register $44_{16}$. [4]

\section{EQUAL 3D ARRAY OF Altitude Wind}

After calculating values of WSP_vyp and WDI_vyp we get a data file from which we can reconstruct a point in the area with the information about wind speed and wind direction. Nevertheless the measured data are unevenly distributed over the analyzed area due to the fact that the data are available only for the point in space where some aircraft is present. On the other hand there may coexist more than one different data for approximately same point in space. On that ground to creating equal 3D array of altitude wind, the steps A and B (described below) had to be done.

\section{A. Fragmentation of the airspace}

For the purposes of fragmentation of the airspace is necessary to split the Czech Republic airspace in horizontal and vertical planes.

There is made a basic net of 18 squares in horizontal plane. Each square has the size of $1.25^{\circ}$ longitude (E) and $1.25^{\circ}$ latitude $(\mathrm{N})$ which covers whole airspace of the Czech Republic. Square area dimensions correspond with square sizes used by WAFC because of possibility of comparison between calculated and forecasted data. In the vertical plane the airspace is divided by FL in the same way as in WAFC (overall 14 vertical planes with different size of FL).

The result of proportioning is dividing airspace into 252 cuboids $(18 \times 24=252)$ that covers whole airspace of the Czech Republic from FL25 to FL600. Dividing of airspace is furthermore differentiating according to parameter DATUM by 1 hour. This way we get imaginary space-time data matrix (basic data matrix) with 6048 cells $(252 \times 24=6048)$ into which we can upload individual records from SSR and their calculated values of wind speed and direction (WSP_vyp, WDI_vyp).

\section{B. Creation of statistical indicators}

Common statistical functions were used over the set of data (WSP_vyp and WDI_vyp) belong to particular cells. Basic measures or indicators used in descriptive statistics were calculated as a mean value $\mu$, variance $\sigma^{2}$ and a standard deviation $\sigma$.

Using the steps $A$ and $B$ on the data file we obtain equal 3D array of altitude wind, which has a matrix data structure. The values in data matrix can be represented in 2 ways - text form or graphical output. The above mentioned operations were again performed in mathematical software MATLAB.

\section{RESULTS}

The calculation of WSP_vyp and WDI_vyp is done by parameters from BDS registers $50_{16}$ (TRK, GSP, TAS) and 6016 (MGH) from SSR data. The calculated values can be directly verified with the values of WSP and WDI recorded by 
aircrafts in BDS register $44_{16}$. The forecasted data from the WAFC are used as another source to evaluate the calculated values. The final evaluation method is to compare the graphical output of the calculated data in MATLAB with meteorological maps from American archives Plymouth State Weather Center.

\section{A. Evaluation according to BDS register $44_{16}$}

The method of evaluation is based on comparison of calculated WSP_vyp, WDI_vyp values with WSP, WDI from BDS register $44_{16}$. The evaluation can be done only if the record contains information about WSP and WDI. In the data file obtained from ANS of the Czech Republic only $5 \%$ (average 75000 records per day) contains information about WSP and WDI.

\section{1) WSP_vyp and WSP}

Table 1 describes a level of equality between WSP_vyp values and WSP in the intervals of accuracy for the data file from the period 01.6.2013-15.6.2013.

TABLE I. ACCURACY OF THE CALCULATION WIND SPEED

\begin{tabular}{|c|r|r|r|r|r|r|r|}
\hline \multicolumn{7}{|c|}{ Percentage equivalence of WSP and WSP_vyp } \\
\hline & \multicolumn{7}{|c|}{ Interval of accuracy } \\
\hline Date & $\mathbf{0 - 1} \mathbf{~ m} / \mathbf{s}$ & $\mathbf{1 - 5} \mathbf{~ m} / \mathbf{s}$ & $\mathbf{5 - 1 0} \mathbf{~ m} / \mathbf{s}$ & $\mathbf{1 0 - 1 5} \mathbf{~ m} / \mathbf{s}$ & $\mathbf{1 5 - 2 0} \mathbf{~ m} / \mathbf{s}$ & $\mathbf{2 0 - 5 0} \mathbf{~ m} / \mathbf{s}$ & $>\mathbf{5 0} \mathbf{~ m} / \mathbf{s}$ \\
\hline $\mathbf{1 . 6 . 2 0 1 3}$ & 44,52 & 50,36 & 5,07 & 0,05 & 0,00 & 0,00 & 0,00 \\
\hline $\mathbf{2 . 6 . 2 0 1 3}$ & 47,20 & 50,25 & 2,36 & 0,19 & 0,00 & 0,00 & 0,00 \\
\hline $\mathbf{3 . 6 . 2 0 1 3}$ & 46,25 & 49,68 & 4,01 & 0,05 & 0,00 & 0,00 & 0,00 \\
\hline $\mathbf{4 . 6 . 2 0 1 3}$ & 43,46 & 52,32 & 4,02 & 0,18 & 0,02 & 0,00 & 0,00 \\
\hline $\mathbf{5 . 6 . 2 0 1 3}$ & 45,11 & 49,22 & 5,41 & 0,26 & 0,00 & 0,00 & 0,00 \\
\hline $\mathbf{6 . 6 . 2 0 1 3}$ & 47,15 & 49,34 & 3,25 & 0,23 & 0,02 & 0,00 & 0,00 \\
\hline $\mathbf{8 . 6 . 2 0 1 3}$ & 45,38 & 53,00 & 1,40 & 0,20 & 0,01 & 0,00 & 0,00 \\
\hline $\mathbf{9 . 6 . 2 0 1 3}$ & 49,91 & 47,25 & 2,78 & 0,05 & 0,00 & 0,00 & 0,00 \\
\hline $\mathbf{1 0 . 6 . 2 0 1 3}$ & 43,38 & 51,66 & 4,89 & 0,07 & 0,00 & 0,00 & 0,00 \\
\hline $\mathbf{1 1 . 6 . 2 0 1 3}$ & 46,89 & 48,56 & 4,19 & 0,28 & 0,07 & 0,00 & 0,00 \\
\hline $\mathbf{1 2 . 6 . 2 0 1 3}$ & 42,52 & 51,93 & 5,22 & 0,32 & 0,02 & 0,00 & 0,00 \\
\hline $\mathbf{1 3 . 6 . 2 0 1 3}$ & 48,05 & 47,55 & 4,28 & 0,12 & 0,00 & 0,00 & 0,00 \\
\hline $\mathbf{1 4 . 6 . 2 0 1 3}$ & 51,29 & 46,01 & 2,36 & 0,31 & 0,01 & 0,00 & 0,00 \\
\hline $\mathbf{1 5 . 6 . 2 0 1 3}$ & 50,83 & 45,50 & 3,62 & 0,05 & 0,01 & 0,00 & 0,00 \\
\hline Total & $\mathbf{4 6 , 5 7 \%}$ & $\mathbf{4 9 , 4 7 \%}$ & $\mathbf{3 , 7 8 \%}$ & $\mathbf{0 , 1 7 \%}$ & $\mathbf{0 , 0 1 \%}$ & $\mathbf{0 , 0 0} \%$ & $\mathbf{0 , 0 0} \%$ \\
\hline
\end{tabular}

In Table 1 the green color indicates volume of calculated values of WSP_vyp with the accuracy of $0-5 \mathrm{~m} / \mathrm{s}$, which is acceptable for ATC purposes so the accuracy is not significantly affect air traffic (adjustment of aircraft's TAS and GSP). Yellow color indicates the volume of WSP_vyp values whose deviation accuracy of $5-20 \mathrm{~m} / \mathrm{s}$ from the real values would be reflected in the planned flight trajectory. Red color indicates the volume of WSP_vyp values with deviation accuracy higher than $20 \mathrm{~m} / \mathrm{s}$ which would have a crucial impact on air traffic.

The results from Table 1 shows that $96.04 \%$ values of WSP_vyp correspond with sufficient accuracy with the values of WSP in BDS register $44_{16} .3 .96 \%$ values of WSP_vyp ranges between permissible deviations values.

\section{2) WDI_vyp a WDI}

Table 2 describes a level of equality between WDI_vyp values and WDI in the intervals of accuracy for the data file from the period 01.6.2013-15.6.2013.
TABLE II. ACCURACY OF THE CALCULATION WIND DIRECTION

\begin{tabular}{|c|r|r|r|r|r|r|r|}
\hline \multicolumn{7}{|c|}{ Percentage equivalence of WDI_vyp and WDI } \\
\hline & \multicolumn{7}{|c|}{ Interval of accuracy } \\
\hline Date & $\mathbf{0}^{\circ} \mathbf{- 5}^{\circ}$ & $\mathbf{5}^{\circ}-\mathbf{1 0}^{\circ}$ & $\mathbf{1 0}^{\circ}-\mathbf{1 5}^{\circ}$ & $\mathbf{1 5}^{\circ}-\mathbf{3 0}$ & $\mathbf{3 0}^{\circ}-\mathbf{5 0}$ & $\mathbf{5 0}^{\circ}-\mathbf{1 0 0}^{\circ}$ & $\mathbf{1 0 0}^{\circ}-\mathbf{1 8 0}^{\circ}$ \\
\hline $\mathbf{1 . 6 . 2 0 1 3}$ & 56,67 & 20,83 & 9,59 & 8,79 & 2,56 & 1,17 & 0,39 \\
\hline $\mathbf{2 . 6 . 2 0 1 3}$ & 56,17 & 19,79 & 9,77 & 9,69 & 2,32 & 1,58 & 0,67 \\
\hline $\mathbf{3 . 6 . 2 0 1 3}$ & 51,29 & 20,85 & 8,88 & 11,64 & 4,20 & 2,18 & 0,98 \\
\hline $\mathbf{4 . 6 . 2 0 1 3}$ & 49,44 & 20,82 & 9,60 & 10,12 & 5,01 & 3,38 & 1,64 \\
\hline $\mathbf{5 . 6 . 2 0 1 3}$ & 31,26 & 18,76 & 12,43 & 19,94 & 9,49 & 5,68 & 2,43 \\
\hline $\mathbf{6 . 6 . 2 0 1 3}$ & 35,38 & 21,55 & 12,67 & 15,70 & 6,62 & 4,93 & 3,13 \\
\hline $\mathbf{8 . 6 . 2 0 1 3}$ & 38,34 & 21,01 & 10,80 & 15,26 & 6,85 & 4,44 & 3,29 \\
\hline $\mathbf{9 . 6 . 2 0 1 3}$ & 53,51 & 21,92 & 9,67 & 9,70 & 2,32 & 1,42 & 1,47 \\
\hline $\mathbf{1 0 . 6 . 2 0 1 3}$ & 34,62 & 19,38 & 11,56 & 16,52 & 8,73 & 6,81 & 2,39 \\
\hline $\mathbf{1 1 . 6 . 2 0 1 3}$ & 34,75 & 19,63 & 11,47 & 15,70 & 8,48 & 6,85 & 3,12 \\
\hline $\mathbf{1 2 . 6 . 2 0 1 3}$ & 59,71 & 17,60 & 7,84 & 8,91 & 2,99 & 1,93 & 1,04 \\
\hline $\mathbf{1 3 . 6 . 2 0 1 3}$ & 52,70 & 22,92 & 10,46 & 8,88 & 2,91 & 1,32 & 0,82 \\
\hline $\mathbf{1 4 . 6 . 2 0 1 3}$ & 67,46 & 17,37 & 8,17 & 4,41 & 0,43 & 1,20 & 0,96 \\
\hline $\mathbf{1 5 . 6 . 2 0 1 3}$ & 67,78 & 19,30 & 8,06 & 3,31 & 0,33 & 0,82 & 0,40 \\
\hline Total & $\mathbf{4 9 , 2 2 \%}$ & $\mathbf{2 0 , 1 2 \%}$ & $\mathbf{1 0 , 0 7 \%}$ & $\mathbf{1 1 , 3 3 \%}$ & $\mathbf{4 , 5 2 \%}$ & $\mathbf{3 , 1 2} \%$ & $\mathbf{1 , 6 2 \%}$ \\
\hline
\end{tabular}

In Table 2 the green color indicates volume of calculated values of WDI_vyp with the accuracy $0^{\circ}-15^{\circ}$, which is acceptable for ATC purposes so the accuracy is not significantly affect air traffic (deviation from the planned flight trajectory and subsequent adjustment of $\mathrm{MGH}$ ). Yellow color indicates the volume of WDI_vyp with deviation of $15^{\circ}-50^{\circ}$ that would be reflected in the planned flight trajectory. Red color indicates the amount of WDI_vyp with deviation accuracy higher than $50^{\circ}$ which would significantly reflect the planned flight trajectory.

The results from Table 2 shows that 79,41\% values of WDI_vyp corresponds with sufficient accuracy with the values of WDI in BDS register $44_{16}$ reported by aircrafts. $15,85 \%$ values of WDI_vyp ranges between permissible deviations values and $4,74 \%$ values of WDI_vyp seems as considerably inaccurate.

\section{B. Graphical evaluation}

Mathematical software MATLAB allows plotting WSP_vyp and WDI_vyp values from space-time data matrix. The plotting method corresponds with classic visualization of wind vector in meteorological maps where the wind direction is illustrated by directional arrow (direction from which the wind blows) and the wind speed is represented by the length of the arrow.

In Figure 1 is meteorological map from the American archive Plymouth State Weather Center. In Figure 2 is graphical output in MATLAB of altitude wind field for 01.06.2013 11:00:00-12:00:00 and FL350-375.

In the area of the Czech Republic we can approved sufficient conformity by means of comparison of the graphical output of calculated values (WSP_vyp and WDI_vyp) in Figure 2 with real values of wind speed and direction shown in a meteorological map from American archive Plymouth State Weather Center. [5] 


\section{Plymouth State Weather Center V}

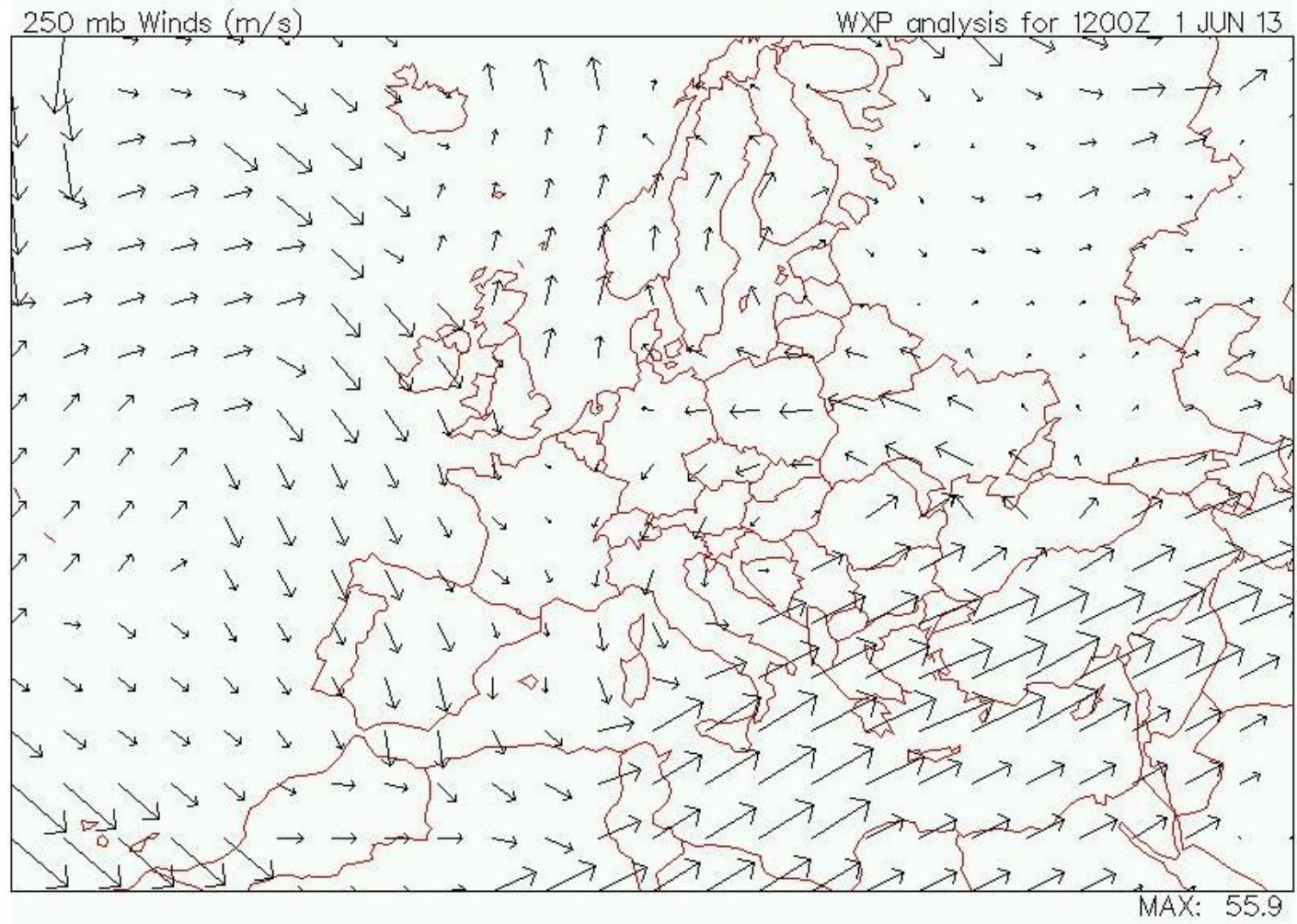

Figure 1. Meteorological map of wind from Plymouth State Weather Center

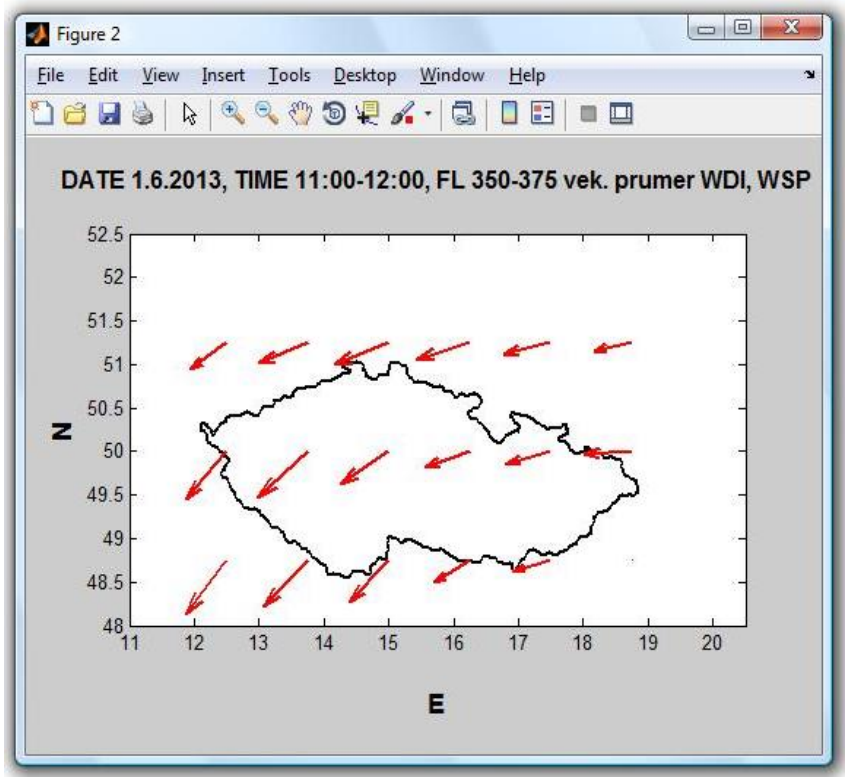

Figure 2. Graphical output of WSP_vyp, WDI_vyp from software MATLAB

\section{Evaluation according to WAFC}

The forecast data for wind from WAFC are stored in the form of cells, whose parameters correspond to the cells in the space-time data matrix created in MATLAB $\left(1.25^{\circ} \times 1.25^{\circ} \mathrm{x}\right.$ FL). The principle of evaluation consists in comparing the calculated values of WSP_vyp and WDI_vyp with values of WSP and WDI from WAFC.

The method of calculating WSP_vyp and WDI_vyp values for individual cells is based on the principle that from all of the records belonging to a specific cell is first calculated wind vector components $\mathrm{Vx}, \mathrm{Vy}$ in Equations (3.1), (3.2), and then their mean value, variance and standard deviation. In the following step the relevant WSP_vyp and WDI_vyp values are calculated in Equations (3.3), (3.4) from the statistical indicators of vector components $\mathrm{Vx}, \mathrm{Vy}$. The mean values of WSP_vyp and WDI_vyp express the representative value of altitude wind field of all records belonging to a specific cell. The method of calculating WSP_vyp and WDI_vyp using the mean value of vector components $\mathrm{Vx}, \mathrm{Vy}$ treats problems of statistical computing operations with values near the border of $0^{\circ} / 360^{\circ}$. 


\section{1) WSP_vyp and WSP}

In Table 3 is shown matrix of divergency of WSP_vyp and WSP values from WAFC data. Cells in the matrix are described with midpoints of parameters $\mathrm{N}$ and $\mathrm{E}$ according to their geographical location.

TABLE III. MATRIX OF DIVERGENCY WSP VYP FROM WSP

\begin{tabular}{|c|r|r|r|r|r|r|}
\hline \multicolumn{7}{|c|}{ Matrix of cells for 4.4.2014 9:00-12:00 FL350-375 } \\
\hline & \multicolumn{7}{|c|}{ Divergency of WSP_vyp and WSP [m/s] } \\
\hline $\mathbf{N ~ 5 1 , 2 5 ^ { \circ }}$ & 2,73 & 0,39 & 0,69 & 1,38 & 0,36 & 4,57 \\
\hline $\mathbf{N ~ 5 0 ^ { \circ }}$ & 0,36 & 0,15 & 1,03 & 1,19 & 4,23 & 0,59 \\
\hline $\mathbf{N ~ 4 8 , 7 5 ^ { \circ }}$ & 1,47 & 1,09 & 0,04 & 3,08 & 2,36 & 2,65 \\
\hline & $\mathbf{E ~ 1 2 , 5 ^ { \circ }}$ & $\mathbf{E ~ 1 3 , 7 5 ^ { \circ }}$ & $\mathbf{E ~ 1 5 ^ { \circ }}$ & $\mathbf{E ~ 1 6 , 2 5 ^ { \circ }}$ & E 17,5 & E 18,75 \\
\hline
\end{tabular}

The mean value of divergency of wind speed is $1.57 \mathrm{~m} / \mathrm{s}$ which ranges according to ATC criteria in acceptable interval of wind speed accuracy $0-5 \mathrm{~m} / \mathrm{s}$ and is satisfactory for purposes ATC. From results in Table 3 can be claimed that values of WSP_vyp in significant volume corresponds with values of WSP from forecasted data WAFC.

\section{2) WDI_vyp and WDI}

In Table 4 is shown matrix of divergency of WDI_vyp and WDI values from WAFC data. Cells in the matrix are described with midpoints of parameters $\mathrm{N}$ and $\mathrm{E}$ according to their geographical location.

TABLE IV. MATRIX OF DIVERGENCY WDI_VYP FROM WDI

\begin{tabular}{|c|r|r|r|r|r|r|}
\hline \multicolumn{7}{|c|}{ Matrix of cells for 4.4.2014 9:00-12:00 FL350-FL375 } \\
\hline & \multicolumn{7}{|c|}{ Divergency of WDI_vyp and WDI [ $\left.{ }^{\circ}\right]$} \\
\hline $\mathbf{N ~ 5 1 , 2 5 ^ { \circ }}$ & 3,53 & 15,38 & 1,00 & 1,71 & 2,12 & 17,62 \\
\hline $\mathbf{N ~ 5 0}$ & 3,01 & 12,72 & 5,29 & 7,60 & 3,80 & 7,23 \\
\hline $\mathbf{N ~ 4 8 , 7 5 ^ { \circ }}$ & 32,98 & 30,63 & 10,19 & 5,09 & 3,72 & 9,06 \\
\hline & $\mathbf{E ~ 1 2 , 5 ~}^{\circ}$ & $\mathbf{E ~ 1 3 , 7 5 ^ { \circ }}$ & $\mathbf{E ~ 1 5}^{\circ}$ & $\mathbf{E ~ 1 6 , 2 5 ^ { \circ }}$ & E 17,5 & $\mathbf{E ~ 1 8 , 7 5}^{\circ}$ \\
\hline
\end{tabular}

The mean value of divergency of wind direction is $9.59^{\circ}$ which ranges according to ATC criteria in acceptable interval of wind direction accuracy $0^{\circ}-15^{\circ}$ and is satisfatory for purposes ATC.

In two cases the divergency is higher than $30^{\circ}$. The reason could be value distortion of some parameters TRK, GSP, MGH, TAS due to overlapping signals on input to SSR from aircraft transponders. Another reason for different calculated values of WDI_vyp could be in differential changes in parameters TRK, GSP, MGH, TAS during aircraft maneuvers (climbing/descending, yawing).

The overall picture of calculating wind speed and direction however largely coincides with the WAFC data with sufficient accuracy so we can the method of calculation consider as applicable. According to the results in Tables 3 and 4, we can also say that greater accuracy of the calculated values are achieved for wind speed because wind direction has characteristics of dynamic variable which can change quickly depending on the surrounding environment.

\section{CONCLUSION}

According to the results of the practical part this article we can claim that we have managed to develop the method which allows to reconstruct information about wind speed and direction for any point in the airspace of the Czech Republic. The accuracy of calculation method was validated on real data from BDS register $44_{16}$ and overall $96 \%$ calculated values of WSP_vyp coincided with WSP with acceptable deviation 0-5 $\mathrm{m} / \mathrm{s}$ and $80 \%$ WDI_vyp coincided with WDI with acceptable deviation $0^{\circ}-15^{\circ}$.

The accuracy of the calculating method affect the input parameters TRK, GSP, MGH, TAS obtained from SSR and the value of the magnetic declination in Equations (3.1), (3.2). The magnetic declination was set to an average value of $2.6^{\circ}$ for the whole area of the Czech Republic. To achieve greater accuracy in the calculation method would be appropriate to use variable value of magnetic declination depending on geographical location.

The achieved results show that the values of altitude wind field are able to calculate with acceptable precision from SSR data. But on the other hand the measurements show that the wind as physical quantity has dynamic characteristics and its values can change very quickly.

Applying the results could lead into creating a suitable tool for data archiving with information about wind speed and direction, which enables its interpretation in graphic or text output. Calculated values of altitude wind field can also be used as a data source of information about wind for different meteorological models. This new approach could improve individual calculating and filtering algorithms or completely show new possibilities how to calculate wind speed and direction from SSR data.

\section{REFERENCES}

[1] ICAO Annex 10 Vol. III

[2] International Civil Aviation Organization. Manual on Mode S Specific Services, Second Edition. Quebec: 2004. Document 9688, AN/952.

[3] Michael J. Hernandez, John L. Viescas. Myslime v jazyku SQL, tvorba dotazů. Praha, Grada Publishing, a.s., 2004.

[4] B.J. Puntin, T.B. Bilingsley, V.M. Heinz, V.A. Orlando, R.T. Wiken. Aircraft Derived Data Algorithms. Lexington: Massachusetts Institute of Technology, Lincoln Laboratory, 2002.

[5] Plymouth State University. Plymouth State Weather Center, Whole Sounding Data Upper Air Maps. Plymouth: 2014. 